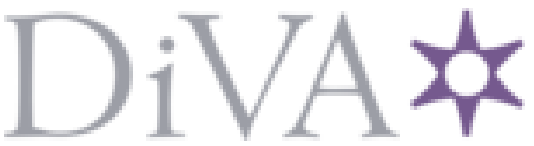

http://www.diva-portal.org

Postprint

This is the accepted version of a paper presented at IEEE 85th Vehicular Technology Conference VTC2017-Spring, o4 Jun 2017, Sydney, Australia.

Citation for the original published paper:

Gutiérrez Peón, P., Uhlemann, E., Steiner, W., Björkman, M. (2017)

Applying Time Diversity for Improved Reliability in a Real-Time Heterogeneous MAC

Protocol

In: 2017 IEEE 85TH VEHICULAR TECHNOLOGY CONFERENCE (VTC SPRING), 8108663

https://doi.org/10.1109/VTCSpring.2017.8108663

N.B. When citing this work, cite the original published paper.

Permanent link to this version:

http://urn.kb.se/resolve?urn=urn:nbn:se:mdh:diva-37002 


\title{
Applying Time Diversity for Improved Reliability in a Real-Time Heterogeneous MAC Protocol
}

\author{
Pablo Gutiérrez Peón*†, Elisabeth Uhlemann ${ }^{\dagger}$, Wilfried Steiner*, and Mats Björkman ${ }^{\dagger}$ \\ *TTTech Computertechnik AG, Vienna, Austria \\ ${ }^{\dagger}$ School of Innovation, Design and Engineering, Mälardalen University, Västerås, Sweden \\ Email: \{pablo.gutierrez-peon, wilfried.steiner\}@tttech.com, \{elisabeth.uhlemann, mats.bjorkman\}@mdh.se
}

\begin{abstract}
Supporting real-time applications over wireless networks is challenging for several reasons. The medium access control (MAC) layer has a key role in enabling real-time guarantees by providing bounded access time to the transmission medium. Unfortunately, even when timely access is granted, transmissions can still be compromised, specially in the wireless domain, due to the unreliable transmission medium. A common way to increase the reliability of a communication system is to apply redundancy in the form of time diversity, i.e., transmitting several copies of the same message at different points in time. In this paper we propose a wireless MAC protocol capable of handling traffic with different levels of criticality and where transmissions and retransmissions are tailored to deadlines, so that the reliability of the communication system is increased and real-time guarantees can be provided. We focus on the traffic scheduling problem, and extend an already existing solution, Time-Triggered Ethernet, suitable for real-time traffic over wired multi-hop networks. The extension enables transmission of heterogeneous traffic over hybrid wired-wireless networks, and provides timely retransmissions in the wireless segment, such that the real-time guarantees are met. Further, we are able to schedule traffic to better cope with interference patterns of different duration, frequency and level of persistence. The results show that the MAC protocol can be successfully applied to combat different kinds of interference while meeting real-time deadlines.
\end{abstract}

\section{INTRODUCTION}

In networking scenarios like industrial automation, realtime communication facilities are mainly provided by wired communication technologies. Even though wired communications is generally better at coping with interference, noise or pathloss, possibilities enabled by wireless communications like reduced wiring, more flexible network deployments, or mobility, are still preferred in many application domains. At the same time, a growing number of devices equipped with sensing and actuation capabilities are now able to communicate over the Internet, a trend that is coined Internet of Things (IoT). IoT brings also an increasing interest in the convergence of real-time network technologies like PROFIBUS, CAN or WirelessHART typically found in the operational technology (OT) field [1], where requirements are imposed by the need to react to physical world processes that evolve over time; and technologies such as Ethernet and WiFi, coming

The research leading to these results has received funding from the People Programme (Marie Curie Actions) of the European Union's Seventh Framework Programme FP7/2007-2013/ under REA grant agreement 607727 (RetNet), 610640 (DREAMS) and 692529 (ECSEL Joint Undertaking), and from National funding through the SafeCOP-project. Björkman and Uhlemann are partly funded by the Knowledge Foundation through READY. from the information technology (IT) application area, where throughput and low cost are favoured. An example of this convergence is Time-Triggered Ethernet (TTE) [2], a wired communication technology based on switched Ethernet that supports traffic with diverse time and safety requirements through three different traffic classes: time-triggered (TT), for applications requiring periodic transmissions with jitter in the range of a few microseconds; rate-constrained (RC), for traffic characterised by an average bandwidth not necessarily periodic; and best-effort (BE), for non real-time traffic. A wireless extension of TTE can play an important role in the IoT ecosystem, by bringing the advantages of a switched Ethernet-based solution, e.g. high throughput and multi-hop support, while at the same time supporting applications with diverse real-time and safety requirements.

A reasonable step for enabling wireless capabilities with TTE is to base it on the IEEE 802.11 standard, since it is similar to Ethernet and offers high throughput. Well-known real-time solutions in industry based on IEEE 802.15.4 and applied in wireless sensor networks (WSN) (e.g. WirelessHART) are not considered in this paper as low energy consumption is less critical in systems with data traffic which also includes $\mathrm{BE}$, and instead high throughput is desired.

In communication systems, the medium access control (MAC) layer is in charge of providing access to the transmission medium. A MAC layer in a real-time network has the additional task of providing this access in a bounded amount of time to prevent deadlines from being missed. Our previous work [3] focused on the MAC layer as a key component to support TT, RC and BE traffic for the wireless extension of TTE over IEEE 802.11. The reason is that the contentionbased access method offered by IEEE 802.11 is not suitable for real-time, and the additional included mechanisms point coordination function (PCF) and hybrid coordination function controlled channel access (HCCA) are based on polling, which introduces a considerable protocol overhead. None of the MAC protocols suggested in the literature for WSN is directly applicable for this use case, since they are not able to cover all requirements. For example, the protocol in [4] is suitable only for BE traffic as it does not avoid two nodes transmitting at the same time. Similarly, the work in [5] is based on overprovisioning transmissions which reduces throughput, but still collisions may occur. In [6], transmissions are constrained to happen from a set of predefined transmitters to a set of 
receivers, restricting other interactions. Further, all messages have to be generated at the same time, and they all share a common deadline. To overcome these limitations, our work used a time division multiple access (TDMA) MAC, in where time-slots are placed on top of the contention-based IEEE 802.11 MAC, and we pre-scheduled a subset of these slots for TT and RC traffic. However, even if timely channel access is granted this way, wireless transmissions are still subject to errors due to fading, which can lead to deadline misses. Hence, in this paper we propose to use time diversity to increase the reliability of the wireless system, so that it is able to keep realtime guarantees under different interference scenarios. Traffic scheduling becomes crucial to bring together the requirements of TT and RC traffic and increased reliability. As of today, the wired version of TTE creates a schedule for TT and RC traffic in multi-hop wired settings. This paper extends the scheduler so it can handle traffic in wireless links, enabling hybrid wiredwireless setups, and also incorporating retransmissions to increase the reliability. Since wireless interference can appear with different levels of intensity, duration, and persistence, we propose different retransmission schemes tailored to the interference pattern. Depending on the burst size, retransmissions can either happen immediately to minimize jitter, or, when the deadline allows, be scheduled to happen slightly later to avoid a temporary outage.

The remainder of the paper is structured as follows. In Section II we present some background on the problem of interference in wireless networks and related work on how retransmissions are used to solve it in solutions based on IEEE 802.11 and IEEE 802.15.4. Section III describes our proposed MAC, whereas Section IV covers the simulations and the analysis of the results under different interference scenarios. We conclude with Section V.

\section{BACKGROUND AND RELATED WORK}

Anastasi et al. [7] investigate the reasons for low reliability in IEEE 802.15.4 and discover that the problem is mainly caused by the contention-based MAC protocol. The authors show that adjusting the contention-based MAC parameter settings, better delivery ratios can be achieved, even in scenarios with several sources of interference. Similarly, Seno et al. [8] state that the backoff procedures and MAC layer retransmissions included in IEEE 802.11 increase the reliability but are not suitable for real-time since they do not meet any time boundaries. Finally, Lo Bello et al. [9] show that interference in IEEE 802.15.4 is not restricted to overlapping channels, but can also be caused by adjacent channels.

The use of retransmissions is a commonly found countermeasure in the literature. In this context, the work by Dominguez-Jaimes et al. [10] focuses on characterizing the use of retransmissions in IEEE 802.11 for industrial channels with a considerable level of interference. Their results show that in general only one retransmission is enough, and that it should be performed immediately after the transmission. The authors also show that smaller messages result in fewer retransmissions. The effects of retransmissions with respect to the addi- tional requirements they impose on real-time traffic are also investigated in literature, with special focus on the scheduling problem. Jonsson et al. [11] perform a real-time schedulability analysis to guarantee that retransmissions are made without any deadline misses in scenarios with error rates that are typically experienced in wireless communications. Retransmissions are allowed as long as the deadline is not reached, and given that they do not compromise other traffic flows. Willig et al. [12] address the problem of increasing reliability through retransmissions in a TDMA protocol that includes relaying nodes that convey information to a central node, by comparing several scheduling options based on heuristics. Demarch et al. [13] deal with the scheduling problem by providing a probabilistic provisioning of retransmissions, with each node calculating the number of retransmissions that is needed for the given channel error model, and sending the requirements in a message to the scheduler. Retransmissions can happen either immediately or be postponed. Similarly, Parsch et al. [14] present a MAC protocol for WSN in where the number of retransmissions is calculated based on the probability of interference and the desired reliability level. Unfortunately, the scheduling solutions proposed in [11]-[14] are wirelessonly and do not operate in the context of multi-hop hybrid wired-wireless networks.

To the best of our knowledge, our proposed protocols are the first to consider a heterogeneous multi-hop wired-wireless network with support for three traffic classes with different timing and safety requirements, in which retransmissions are scheduled to meet real-time deadlines, and protocol parameters can be tuned to cope with different interference scenarios.

\section{MAC PRotocol WITH INCREASED RELIABILITY FOR TIME AND SAFETY CRITICAL TRAFFIC}

\section{A. Network model and scheduling}

The design of our MAC protocol intended for wireless communications considers the three different traffic classes from TTE: TT, RC and BE. The characterization of the traffic is as follows: TT is periodic, and defined by its period $t_{\text {period }}$, and data length $d_{\text {length }}$. RC is determined by the minimum interarrival time between the messages $t_{M I A}$, and $d_{\text {length }}$. Finally, BE is defined only by $d_{\text {length }}$. Further, the physical topology of the multi-hop network is given by the undirected graph $G(V, E)$, where $V$ stands for the network nodes, and $E$ represents the physical links between the nodes. In our network, every physical link is bi-directional and defines two directed dataflow links, with the set of them being $L$, so that

$$
\forall\left[v_{1}, v_{2}\right] \in V:\left(v_{1}, v_{2}\right) \in E \Rightarrow\left[v_{1}, v_{2}\right],\left[v_{2}, v_{1}\right] \in L .
$$

In order to combine the different types of traffic under a single communication infrastructure, an offline schedule is created for all the TT traffic flows, so that every hop of a message between the network devices is scheduled. TTE is based on TDMA, and the nodes are given channel access opportunities in the form of time-slots. For simplicity, we model RC traffic also as periodic and treat it in the same way as TT traffic, so that $t_{\text {period }}=t_{M I A}$. This assumption 
guarantees RC traffic even in the worst case, and assures that the largest time waiting for a RC slot is not greater than $t_{M I A}$. As in the case of other TDMA-based protocols, a common notion of the time in all the nodes is necessary. This is normally achieved with synchronization protocols that introduce a limited amount of overhead. A straightforward way to manage this overhead is by mapping it to the TT traffic class. Once we get the schedule, we proceed in the same way TTE does and distribute it among the nodes prior to operation. After TT and RC traffic is allocated, the remaining time can be claimed dynamically by other non-critical traffic (e.g. BE).

A TT or RC message $m$ that needs to be transmitted from a sender to a receiver is decomposed in a set of message instances $m^{\left[v_{i}, v_{j}\right]}$, with every instance being transmitted in one dataflow link $\left[v_{i}, v_{j}\right]$ from the set of dataflow links $L$. The network schedule includes the instances of all messages, defined by $m^{\left[v_{i}, v_{j}\right]}=\left\{m . t_{\text {period }}, m \cdot d_{\text {length }}, m^{\left[v_{i}, v_{j}\right]} \cdot t_{\text {offset }}\right\}$. Further, $t_{\text {offset }}$ specifies the transmission time of the instance of a message and has to be calculated. The task of calculating $t_{\text {offset }}$ is performed by the TTE scheduler, that is based on the use of first-order logic constraints to model the network topology and traffic flows [15]. The capacity of the firstorder logic constraints to model complex networking scenarios with simple mathematical expressions, and the faculty to be combined in a scalable way, are among the main reasons to use this tool for scheduling on TTE. The existing TTE scheduler handles several constraints, but for the purpose of this paper we focus on the avoid-collision, that prevent the messages instances to be placed concurrently in the same dataflow link.

When supporting wireless communication, we also require to avoid concurrent transmissions on all wireless links within range of each other. We therefore redefine $L$, so that it is now composed of both wired $L_{w d}$ and wireless dataflow links $L_{w l}$ : $L=L_{w d} \cup L_{w l}$. For simplicity, we assume that the slot size is the same for both the wired and wireless segments. Given the set of messages $M$, and $L C M\left(M . t_{\text {period }}\right)$ standing for the least common multiple of all message periods (harmonic periods), the following equation complements the wired-only version from [15] to additionally enable wireless links:

$$
\begin{aligned}
& \forall\left[v_{k}, v_{l}\right],\left[v_{q}, v_{r}\right] \in L, \forall m_{i}, m_{j} \in M, \\
& \forall a \in\left[0,1, \ldots,\left(\frac{L C M\left(M \cdot t_{\text {period }}\right)}{m_{i} \cdot t_{\text {period }}}-1\right)\right], \\
& \forall b \in\left[0,1, \ldots,\left(\frac{L C M\left(M \cdot t_{\text {period }}\right)}{m_{j} \cdot t_{\text {period }}}-1\right)\right]: \\
& \left(\left(m_{i} \neq m_{j}\right) \wedge \exists m_{i}^{\left[v_{k}, v_{l}\right]} \wedge \exists m_{j}^{\left[v_{q}, v_{r}\right]}\right. \\
& \left.\wedge\left(\left[v_{k}, v_{l}\right] \in L_{w l} \wedge\left[v_{q}, v_{r}\right] \in L_{w l}\right)\right) \Rightarrow \\
& \left(\left(a \times m_{i} \cdot t_{\text {period }}\right)+m_{i}^{\left[v_{k}, v_{l}\right]} \cdot t_{\text {offset }} \neq\right. \\
& \left.\left(b \times m_{j} \cdot t_{\text {period }}\right)+m_{j}^{\left[v_{q}, v_{r}\right]} \cdot t_{\text {offset }}\right) .
\end{aligned}
$$

In order to find a solution that satisfies all the constraints imposed, satisfiability modulo theories (SMT) solvers [16] are used [15]. Creating a schedule is an offline task that does not introduce overhead in the runtime operation of the MAC protocol. With the definition of the constraints above, the MAC protocol is provided with a schedule that avoids the problem of interference from traffic inside the network. Still, the problem of external interference remains to be solved.

\section{B. Retransmission schemes}

We schedule retransmissions to increase the reliability of TT and RC traffic. To increase the reliability of TT traffic, the period can be reduced (e.g., by oversampling the sensor), such that some lost transmissions can be tolerated. Alternatively, if the TT data is used for majority voting for increased safety, retransmissions made before the deadline expires are tractable. $\mathrm{RC}$ traffic can be used for event-driven data, in which case timely retransmission for increased reliability is desirable. To schedule retransmissions in addition to the original transmissions, it is sufficient to add a constraint to the scheduler that forces the allocation of one or several retransmissions after the first scheduled transmission. Given the desired number of retransmissions $R$, and the inter-transmission time $t_{I T I}$, as the interval between a transmission and its matching retransmission, next equation adds retransmissions to Equation 2:

$$
\begin{aligned}
& \forall\left[v_{k}, v_{l}\right],\left[v_{q}, v_{r}\right] \in L, \forall m_{i}, m_{j} \in M, \\
& \forall a \in\left[0,1, \ldots,\left(\frac{L C M\left(M \cdot t_{\text {period }}\right)}{m_{i} \cdot t_{\text {period }}}-1\right)\right], \\
& \forall b \in\left[0,1, \ldots,\left(\frac{L C M\left(M \cdot t_{\text {period }}\right)}{m_{j} \cdot t_{\text {period }}}-1\right)\right], \\
& \forall c \in\{0 . . R\} \in \mathbb{N}^{0}: \\
& \left(\left(m_{i} \neq m_{j}\right) \wedge \exists m_{i}^{\left[v_{k}, v_{l}\right]} \wedge \exists m_{j}^{\left[v_{q}, v_{r}\right]}\right. \\
& \left.\wedge\left(\left[v_{k}, v_{l}\right] \in L_{w l} \wedge\left[v_{q}, v_{r}\right] \in L_{w l}\right)\right) \Rightarrow \\
& \left(\left(a \times m_{i} \cdot t_{\text {period }}\right)+m_{i}^{\left[v_{k}, v_{l}\right]} \cdot t_{\text {offset }}+c \times t_{I T I} \neq\right. \\
& \left.\left(b \times m_{j} \cdot t_{\text {period }}\right)+m_{j}^{\left[v_{q}, v_{r}\right]} \cdot t_{\text {offset }}+c \times t_{\text {ITI }}\right) .
\end{aligned}
$$

The jitter in this case is defined as the time difference between the minimum delay that happens if and when the first transmission is successful, and the maximum delay that happens if and when the last allowed retransmission does not succeed (infinite delay). This parameter will be affected both by $R$ and by $t_{I T I}$. Depending on the specific value adopted by $t_{I T I}$, retransmissions can either be close to or further from the original transmission. An important factor to consider when designing retransmission schemes is that interference usually happen in bursts, characterized by a duration $t_{\text {burst }}$. Given this, we propose two retransmission schemes: one scheduling retransmissions right after its corresponding transmission slot, called Consecutive, and another one with spread retransmissions, called Spread. The former is expected to provide a lower jitter, whereas the latter is expected to be better against bursts of interference by increasing the chances of avoiding the burst. Additionally, all nodes even if the current slot is not assigned to them, check if the transmission medium is free for a duration of the turn-around time represented by the interframe space $t_{I F S}$, coming from the physical layer of IEEE 802.11. If the medium is free during $t_{I F S}$, it means that the slot is not used for scheduled traffic and it can now be accessed via contention. 


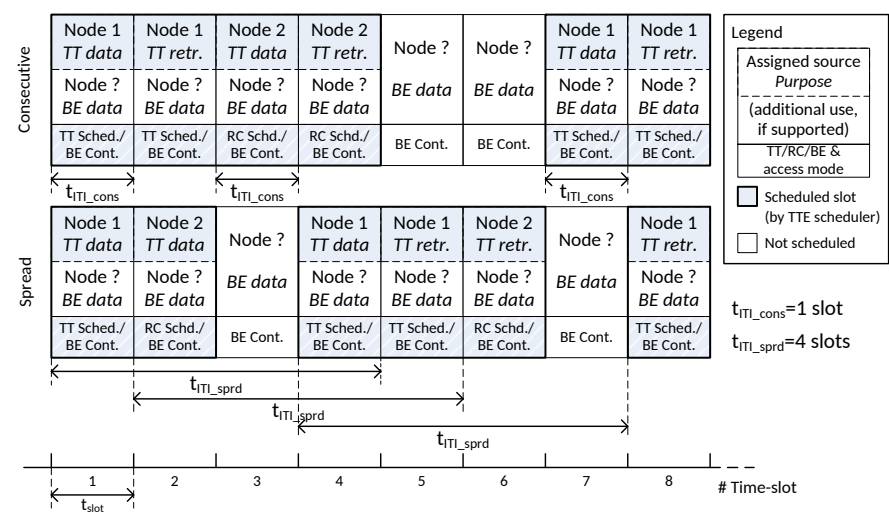

Fig. 1. Proposed retransmission schemes. Note that scheduled slots may be reused by BE traffic when no scheduled traffic is sent by the assigned node.

Consecutive retransmissions. A straightforward way to include retransmissions consist in placing each one consecutively after the first transmission attempt. This can be implemented by making each slot bigger, and allocating both the transmission and the retransmission inside the slot. However, we discard this option, since then the unused retransmission time cannot be reclaimed by other traffic. Hence, we use the mechanism as shown in Figure 1, where the transmission and retransmissions (one in this case), are placed in separated slots. To schedule this scheme, the scheduler will first place the transmission, and then the retransmissions, with a shift between them of the duration of the slot, so that $t_{I T I}$ for consecutive retransmissions becomes $t_{I T I_{-} \text {cons }}=t_{\text {slot }}$.

Spread retransmissions. In the Spread scheme we propose to spread the retransmissions, as shown in Figure 1, so that the $t_{I T I}$ in Spread $t_{I T I_{-} s p r d}$ is larger than in the Consecutive scheme: $t_{I T I \_s p r d}>t_{I T I_{-} \text {cons }}$. The specific value of $t_{I T I_{-} s p r d}$ must be a tradeoff between two factors. On one hand, having as low value as possible so that it is easier to get a schedule given that the traffic flow cannot meet its deadline if the value is too big and a lower value also decreases the transmission jitter. On the other hand, having a large enough value that makes possible to avoid bursts of interference $\left(t_{I T I_{-} s p r d}>t_{\text {burst }}\right)$. In this respect, it is desired to characterize the interference present in the location where the network will be installed, e.g. in [17] authors provide a characterization of industrial wireless channels, so that properties like the maximum $t_{b u r s t}$ can be derived, and the most appropriate value of $t_{I T I}$ can be set in the scheduler. Also, note that although a low jitter is very relevant for TT, it is not necessarily the case for RC, suggesting that different strategies could be used for TT and $\mathrm{RC}$ traffic.

Regarding the value of $R$, it must be chosen by the user in both retransmission schemes, and the specific value depends on the deadline and desired reliability level.

\section{Time-slot details}

The time-slots are the resource that the scheduler allocates based on the imposed constraints. Inside the slot, a single data transmission or retransmission happens. The benefit of using retransmissions is present only when both the following conditions are met: previous transmission attempts have failed, and data is still useful at the time the retransmission is performed, otherwise they become a waste of bandwidth. For this reason, the sender can be informed of the success of a transmission, so that it can decide whether to retransmit or not. These mechanisms based on retransmitting only after receiving feedback are commonly referred as to automatic repeat request (ARQ). The feedback is normally conceived as an acknowledgement message (ACK), sent by the destination upon arrival of a correct message. The information carried by the ACK message can in our case be useful for cancelling further retransmissions and instead using this time for BE traffic or retransmissions from other flows. In systems where there is no such feedback (e.g. simplex communication), retransmissions always take place. To this end, we offer two MAC protocol variations, one without and one with ACK. Both protocol variations use mechanisms already present in IEEE 802.11 and do not increase the complexity of the system. We refer to them as MAC Protocol 1 and MAC Protocol 2 respectively.

In Protocol 1 slots are pre-allocated and retransmissions always take place. The slot size for Protocol $1 t_{\text {slot_mac_1 }}$, needs to account for the time it takes to transmit the data $t_{d a t a}$, and the slot time $t_{S T}$, where $t_{S T}$ is a guard interval used before every transmission to avoid collisions due to propagation delay. The specific value for $t_{S T}$ comes from the IEEE 802.11 physical layer. This makes the slot size for Protocol 1 to be: $t_{\text {slot_mac_1 }}=t_{S T}+t_{\text {data }}$.

Protocol 2 uses feedback so that retransmissions do not take place if the previous message transmission or retransmissions were successful. The advantage of this approach is that the usage of a slot can change dynamically, from being scheduled for retransmissions of critical traffic, to a contention-access slot that can be claimed by nodes that have BE traffic to send. We send the ACK message in the same slot just after the data has been received. Note that if the data was able to arrive, it is likely that the channel is still in good condition also for the ACK, so chances of successful ACK delivery are higher. This in turn implies that the size of the slot in Protocol $2 t_{\text {slot_mac_2 }}$, must be calculated to accommodate the longest of its potential uses: either a prioritized node accesses the slot and uses the feedback mechanism, or the prioritized node does not access the slot and this is accessed via contention. For the first use case, that we refer to as $2 \mathrm{~A}$, the slot must accommodate the $t_{d a t a}$ and $t_{S T}$, as in Protocol 1, plus the ACK $t_{a c k}$, and the turn-around time represented by a short interframe space $t_{S I F S}$, the latter coming from the physical layer of IEEE 802.11. The resulting slot size for $2 \mathrm{~A}$ is $t_{\text {slot_mac_2A }}=t_{S T}+t_{\text {data }}+t_{\text {SIFS }}+t_{a c k}$. For the second use case, $2 \mathrm{~B}$, both $t_{I F S}$ and a contention window to minimize collisions $t_{C W}$ are needed together with the $t_{d a t a}$, resulting in $t_{\text {slot_mac_2B }}=t_{I F S}+t_{C W}+t_{d a t a}$. All in all, the size of a slot for Protocol 2 is calculated as follows:

$$
t_{\text {slot_mac_2 }}=\max \left\{t_{\text {slot_mac_2A }}, t_{\text {slot_mac_2B }}\right\} \text {. }
$$




\section{Simulation and Results}

\section{A. Simulation setup}

To evaluate the resistance of the two MAC protocols and the two proposed retransmission schemes against different interference patterns, we have created a model in OMNeT++ [18], a discrete event C++ library and simulation framework. Specifically, the models are implemented using the INET simulation framework, that in the version we used supports the simulation of the IEEE $802.11 \mathrm{~b}$ physical and MAC layer, but results are also applicable to the standard versions up to the current IEEE 802.11-2012.

The considered performance metrics are percentage of failed transmissions, percentage of time left for $\mathrm{BE}$ traffic, and average delay from MAC layer to MAC layer. Since the scheduler guarantees that retransmissions do not take place after the message deadline, a deadline miss is accounted every time a message is not successfully delivered after the last scheduled retransmission. With the percentage of failed transmissions, we evaluate to what extent TT and RC traffic can follow the schedule under different interference scenarios. The percentage of time left for BE traffic evaluates the differences between Protocol 1 and 2 regarding the time dedicated for BE. This time may differ due to different slot sizes between the protocols, and is subject to the mechanism for reusing slots in Protocol 2. The performance metric average MAC to MAC delay is calculated as the average time it takes from the moment when a message is sent for the first time until the last retransmission attempt is performed (if needed), plus the transmission time. It allows to compare the average delay introduced by the retransmission schemes at MAC level, which grows with every failed transmission attempt.

The setup we have used is based on a small wireless network in infrastructure mode, with five wireless end-systems (WES) and one access point (AP) (Figure 2), which is enough to discern the differences that allow to compare the simulation scenarios. Since the simulation focuses on the effects of interference on real-time traffic on the wireless MAC protocol, we do not simulate BE traffic and the complete hybrid network. Traffic travels from the end-systems to the AP, and is evenly generated. The size of the exchanged packets is 62 bytes (of which 4 bytes are payload), relatively small but representative for the kind of packets typically exchanged in industrial networks. Since we want to support as high transfer rates as possible, we have chosen $11 \mathrm{Mbps}$, the highest offered by $802.11 \mathrm{~b}$. The default values for $t_{S T}=20 \mu \mathrm{s}, t_{S I F S}=10 \mu \mathrm{s}$, and $t_{a c k}=203 \mu \mathrm{s}$ are also taken from the standard.

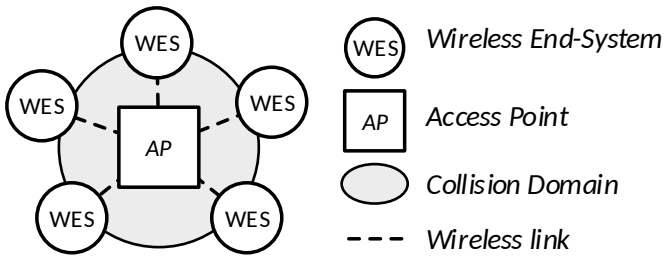

Fig. 2. Simulation scenario.
TABLE I

PROTOCOL RELATED SIMULATION PARAMETERS.

\begin{tabular}{|l|l|}
\hline \multirow{2}{*}{ MAC protocol } & Protocol 1 (no ACK) \\
\cline { 2 - 2 } & Protocol 2 (with ACK) \\
\hline \multirow{2}{*}{ Retransmission scheme } & None \\
\cline { 2 - 2 } & Consecutive \\
\cline { 2 - 2 } & Spread \\
\hline Number of retransmissions $R$ & 1 \\
\hline
\end{tabular}

TABLE II

INTERFERENCE PATTERN RELATED SIMULATION PARAMETERS.

\begin{tabular}{|c|c|}
\hline \multirow{2}{*}{ Type of interference } & CSMA \\
\hline & Jamming \\
\hline \multirow{2}{*}{ Level of interference } & $10 \%$ \\
\hline & $30 \%$ \\
\hline \multirow{3}{*}{$\begin{array}{l}\text { Interference burst size } \\
\text { (as multiple of the size of } \\
\text { data messages) } t_{b u r s t}\end{array}$} & 1 \\
\hline & 15 \\
\hline & 30 \\
\hline
\end{tabular}

We have tested Protocol 1 and 2, for $R=0$ (retransmission scheme None) and $R=1$ (for Consecutive and Spread schemes). We have not set a larger number for $R$, since this shortens the simulation time, while still showing the necessary performance differences, with results that are easily extended to a larger number of retransmissions. Table I offers a summary of protocol related parameters. We have evaluated different channel conditions, including different types of interference: coming from contention-based carrier sense multiple access (CSMA) devices, typical from co-located IEEE 802.11 and IEEE 802.15.4 devices; and jamming interference, with the latter serving to model any other source of unintentional interference. Interference appears at different levels of intensity, being present $10 \%$ and $30 \%$ of the total time. Interference is randomly generated, but can appear in bursts of different sizes, $t_{\text {burst }}$. The size is a multiple of the time it takes to send a packet of 62 bytes. Given these parameters, the worst interference scenario is represented by jamming 30\% of the time, a situation that we consider extreme, i.e., not many protocols, if any, cope with this level of interference, but we use it to test the boundaries of our proposed protocols. Table II offers a summary of interference pattern related parameters. To make results comparable, all the scenarios generate the same amount of traffic, adapted to the protocol that is able to cope with the shortest amount of traffic so that no queue overflows happen. The combination of protocol and interference pattern related parameters results in 72 unique scenarios. We have run the simulations to obtain at least 100 packet losses per scenario for the presented cases.

\section{B. Simulation results}

In Figure 3 and 4, the percentage of failed message transmissions for MAC Protocol 1 and 2 respectively under different loads and burst sizes of CSMA interference is presented. The superposed thinner bars indicate the same performance parameter but for the jamming type of interference. We can see that the jamming type of interference is more harmful than CSMA, given that CSMA devices backoff if they sense the medium as busy. The level of interference causes the expected 
effect, the more time that is occupied by interference, the greater is the deadline miss ratio. However, it can be seen that for the burst size, a larger one does not always mean that results will be worse. This is because with the same level of interference, a larger burst size makes the interference to be constrained to larger, but less frequent interference intervals. From the figures we see that the Consecutive retransmission policy behaves better for the CSMA type of interference, while for the jamming case, the Spread retransmission policy is much more beneficial. Unfortunately, the jamming interference pattern is still very harmful, even with the Spread retransmission policy, but can be improved by increasing the number of retransmissions.

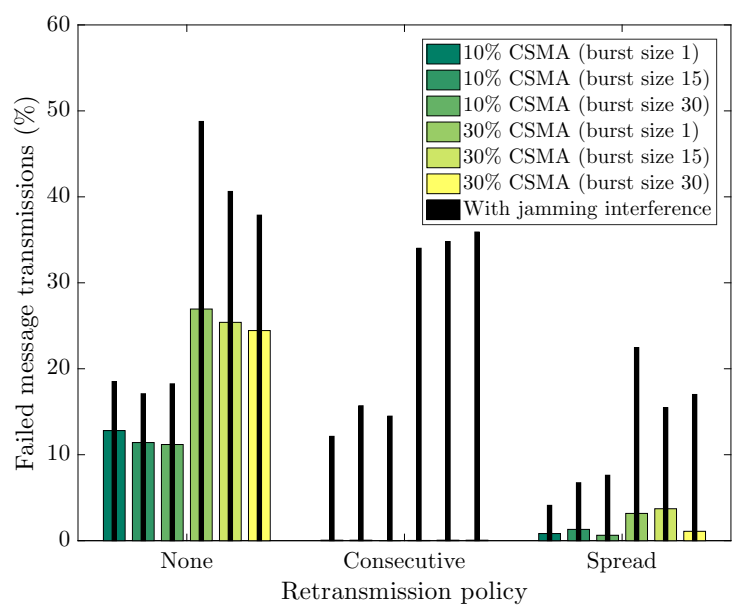

Fig. 3. Percentage of failed message transmissions for MAC Protocol 1 with different loads and burst sizes of CSMA and jamming interference.

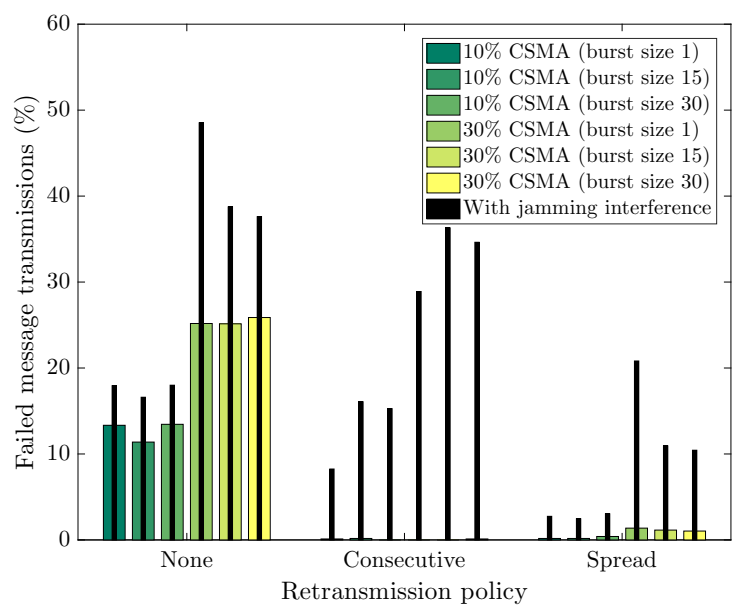

Fig. 4. Percentage of failed message transmissions for MAC Protocol 2 with different loads and burst sizes of CSMA and jamming interference.

Figure 5 and 6 show the percentage of the total time that can be used by BE transmissions in MAC Protocol 1 and 2 respectively under different patterns of CSMA interference. The superposed thinner bars indicate the same performance parameter but for the jamming type of interference. The BE slots are allocated when no retransmissions are scheduled
(None), and also in Protocol 2 when retransmission slots are left free in case the transmission is successful on a previous attempt. In the None case, a big gap between Protocol 1 and 2 can be seen, since Protocol 1 accounts shorter slots and is able to allocate a larger number of them. In this specific example, with small messages being transmitted, the overhead introduced by Protocol 2 is very noticeable, and causes Protocol 1 to allocate almost double the number of slots than Protocol 2. Better performance for Protocol 2 will be observed with an increase in the size of the data.

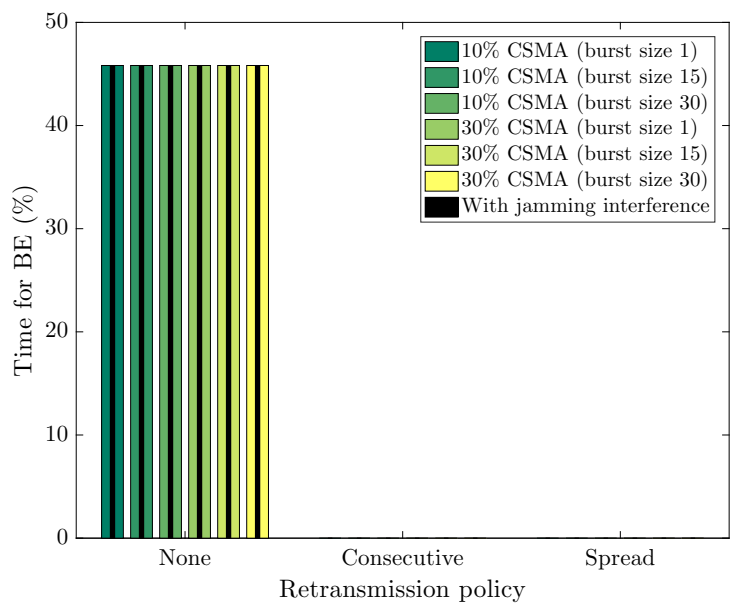

Fig. 5. Percentage of time left for BE transmissions for MAC Protocol 1 with different loads and burst sizes of CSMA and jamming interference.

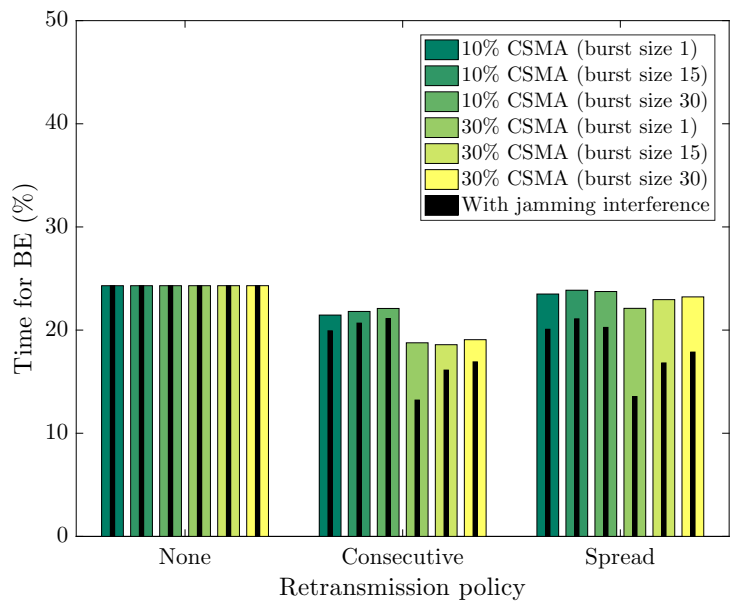

Fig. 6. Percentage of time left for BE transmissions for MAC Protocol 2 with different loads and burst sizes of CSMA and jamming interference.

In Figure 7 and Figure 8, the average MAC to MAC delay under different patterns of CSMA interference is presented for MAC Protocol 1 and MAC Protocol 2 respectively. The superposed thinner bars indicate the same performance parameter but for the jamming type of interference. In both cases, the delay does not grow considerably between the cases of not having a retransmission scheme and the Consecutive scheme. In the case of the Spread scheme, the delay is much larger, since the retransmission slots are more spaced, and increases 
proportionally to the burst size. Note the difference between the average delay for MAC 1 and MAC 2, that is slightly larger for MAC 2 due to the larger slot size required by the protocol overhead.

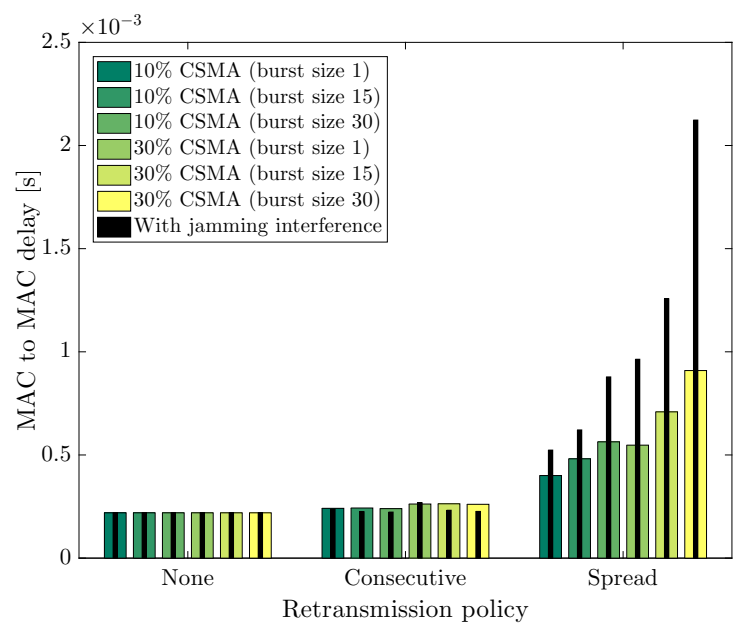

Fig. 7. Average MAC to MAC delay for MAC Protocol 1 with different loads and burst sizes of CSMA and jamming interference.

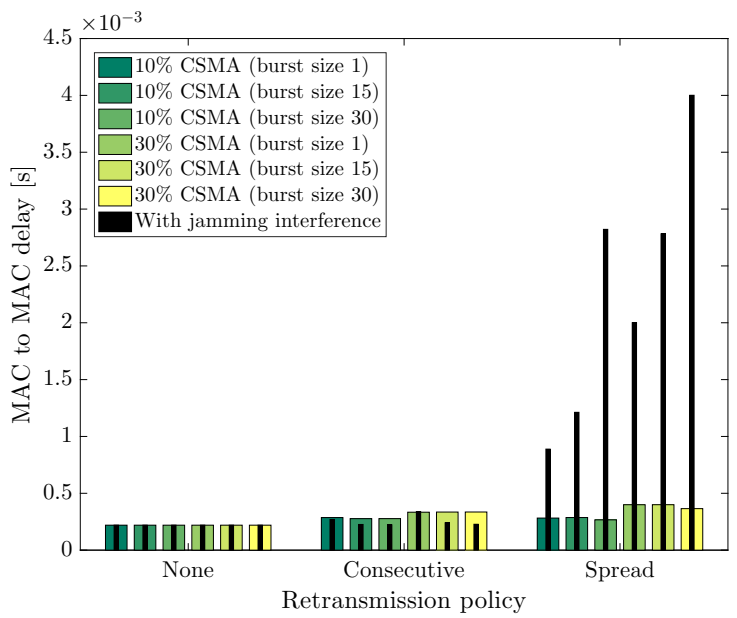

Fig. 8. Average MAC to MAC delay for MAC Protocol 2 with different loads and burst sizes of CSMA and jamming interference.

\section{CONCLUSION}

In this paper we have applied time diversity retransmissions to improve the reliability of a real-time MAC protocol intended to be used in the wireless segment of a heterogeneous wired/wireless network. The protocol is able to support traffic with diverse time and safety requirements, and is designed as an extension of the wired Ethernet-based protocol TTE. We have focused on extending the scheduler of TTE, that already provided a solution for real-time traffic over wired multi-hop networks. The extension allows to support traffic over hybrid wired-wireless networks, and handles both transmissions and retransmissions while keeping real-time guarantees. Two variations of the MAC protocol have been presented, one version making use of all the scheduled retransmissions, and another version based on feedback, that allows to dynamically reuse the retransmission slots that are not needed. Two reliability mechanisms also are applied to these protocols, with retransmissions either happening immediately or postponed. The former minimizes jitter, whereas the latter is better equipped to cope with burst errors. The results show that retransmissions are an effective way to increase the reliability of the communication system, and that the different retransmission schemes can be applied to fit error patterns of different levels of intensity, duration, and persistence.

\section{REFERENCES}

[1] W. Steiner, P. Gutiérrez Peón, M. Gutiérrez, A. Mehmed, G. RodriguezNavas, E. Lisova, and F. Pozo, "Next generation real-time networks based on IT technologies," in Proc. IEEE ETFA, Berlin, Germany, 2016, pp. $1-8$.

[2] W. Steiner, G. Bauer, B. Hall, and M. Paulitsch, "TTEthernet: TimeTriggered Ethernet," in Time-Triggered Communication, R. Obermaisser, Ed. CRC Press, 2011.

[3] P. Gutiérrez Peón, E. Uhlemann, W. Steiner, and M. Björkman, "Medium access control for wireless networks with mixed criticality real-time requirements," in Proc. IEEE IECON, Florence, Italy, 2016, pp. 46654670.

[4] M. R. Palattella, T. Watteyne, Q. Wang, K. Muraoka, N. Accettura, D. Dujovne, L. A. Grieco, and T. Engel, "On-the-fly bandwidth reservation for 6TiSCH wireless industrial networks," IEEE Sensors Journal, vol. 16, no. 2, pp. 550-560, 2016.

[5] S. Duquennoy, B. Al Nahas, O. Landsiedel, and T. Watteyne, "Orchestra: Robust mesh networks through autonomously scheduled TSCH," in Proc. ACM Sensys, Seoul, South Korea, 2015, pp. 337-350.

[6] F. Dobslaw, T. Zhang, and M. Gidlund, "End-to-end reliability-aware scheduling for wireless sensor networks," IEEE Trans. Ind. Informatics, vol. 12, no. 2, pp. 758-767, 2016.

[7] G. Anastasi, M. Conti, and M. Di Francesco, "A comprehensive analysis of the MAC unreliability problem in IEEE 802.15.4 wireless sensor networks," IEEE Trans. Ind. Informatics, vol. 7, no. 1, pp. 52-65, 2011.

[8] L. Seno, S. Vitturi, and F. Tramarin, "Tuning of IEEE 802.11 MAC for improving real-time in industrial wireless networks," in Proc. IEEE ETFA, Krakow, Poland, 2012, pp. 1-8.

[9] L. L. Bello and E. Toscano, "Coexistence issues of multiple co-located IEEE 802.15.4/ZigBee networks running on adjacent radio channels in industrial environments," IEEE Trans. Ind. Informatics, vol. 5, no. 2, pp. 157-167, 2009.

[10] I. Dominguez-Jaimes, L. Wisniewski, H. Trsek, and J. Jasperneite, "Link-layer retransmissions in IEEE 802.11g based industrial networks," in Proc. IEEE WFCS, Nancy, France, 2010, pp. 83-86.

[11] M. Jonsson and K. Kunert, "Towards reliable wireless industrial communication with real-time guarantees," IEEE Trans. Ind. Informatics, vol. 5 , no. 4, pp. 429-442, 2009.

[12] A. Willig and E. Uhlemann, "Deadline-aware scheduling of cooperative relayers in TDMA-based wireless industrial networks," Wireless networks, vol. 20, no. 1, pp. 73-88, 2014.

[13] D. D. Demarch and L. B. Becker, "An integrated scheduling and retransmission proposal for firm real-time traffic in IEEE 802.11e," in Proc. ECRTS, Pisa, Italy, 2007, pp. 146-158.

[14] P. Parsch and A. Masrur, "A reliability-aware medium access control for unidirectional time-constrained WSNs," in Proc. RTNS, 2015, pp. 297-306.

[15] W. Steiner, "An evaluation of SMT-based schedule synthesis for timetriggered multi-hop networks," in Proc. IEEE RTSS, San Diego, USA, 2010, pp. 375-384.

[16] L. De Moura and N. Bjørner, "Satisfiability modulo theories: introduction and applications," Comm. of the ACM, vol. 54, no. 9, pp. 69-77, 2011.

[17] D. Block, H. Tresk, and U. Meier, "Real-Time characterization of fastvarying industrial wireless channels," in Proc. RADCOM, Hamburg, Germany, 2013.

[18] A. Varga, "The OMNeT++ discrete event simulation system," in Proc. ESM, vol. 9, no. S 185, Prague, Czech Republic, 2001, p. 65. 